PROCEEDINGS OF THE

AMERICAN MATHEMATICAL SOCIETY

Volume 129, Number 1, Pages 111-119

S 0002-9939(00)05736-

Article electronically published on August 30, 2000

\title{
REALIZING ALTERNATING GROUPS AS MONODROMY GROUPS OF GENUS ONE COVERS
}

\author{
MIKE FRIED, ERIC KLASSEN, AND YAACOV KOPELIOVICH \\ (Communicated by Michael Stillman)
}

\begin{abstract}
We prove that if $n \geq 4$, a generic Riemann surface of genus 1 admits a meromorphic function (i.e., an analytic branched cover of $\mathbb{P}^{1}$ ) of degree $n$ such that every branch point has multiplicity 3 and the monodromy group is the alternating group $A_{n}$. To prove this theorem, we construct a Hurwitz space and show that it maps (generically) onto the genus one moduli space.
\end{abstract}

\section{IntroduCtion}

Associated to any $n$-sheeted branched cover of $\mathbb{P}^{1}$ with branch set $B \subset \mathbb{P}^{1}$ is a homomorphism $\pi_{1}\left(\mathbb{P}^{1}-B\right) \rightarrow S_{n}$ (the symmetric group) called the monodromy representation of the branched cover. The image of this homomorphism in $S_{n}$ is simply called the monodromy group of the cover (this group is well-defined up to conjugacy in $S_{n}$ ). If $\Sigma$ is a compact Riemann surface and $\phi$ is a nonconstant meromorphic function on $\Sigma$, then $\phi: \Sigma \rightarrow \mathbb{P}^{1}$ is a branched cover and so we may speak of the monodromy group of $\phi$. In [GN], it is stated that "Thompson (private correspondence) has verified that $A_{4}$ is the monodromy group of the generic Riemann surface of genus 1 (as far as we are aware, this is the only known example of a cover of a generic genus $g>0$ surface with monodromy group different from a symmetric group)". Our main result in this paper (Theorem 1, stated formally and proved in Section 4) states that this is true for all $A_{n}$, where $n \geq 4$. More precisely, Theorem 1 asserts that if $n \geq 4$, then a generic Riemann surface of genus one admits a meromorphic function of degree $n$ whose monodromy group is the alternating group $A_{n}$ and all of whose branch points have multiplicity 3 . By generic, we mean that for a given $n$, all but a finite number of genus 1 Riemann surfaces admit such functions.

It also says, the set of Riemann surfaces of genus $g \geq 1$, with functions admitting branch points of multiplicity only 3 , defines an algebraic set of dimension $\geq 1$ in the module space of curves of genus $g$ (Section 4, Comment 0). There is only one Riemann surface of genus one which admits a meromorphic function with monodromy $A_{3}$ : it is the Fermat curve $x^{3}+y^{3}+z^{3}=0$, and the meromorphic function is projection onto any one of the three coordinate axes in $\mathbb{P}^{2}$. To see that there is only one such curve, note that, first, the location of the three branch points in $\mathbb{P}^{1}$

Received by the editors March 8, 1999.

1991 Mathematics Subject Classification. Primary 30F10.

Key words and phrases. Riemann surface, monodromy group, Hurwitz space.

(C)2000 American Mathematical Society 
is irrelevant to the moduli and, second, the combinatorics is completely determined by the monodromy requirements (since the only way to select three 3 -cycles in $A_{3}$ whose product is 1 is to select the same 3 -cycle three times).

We now give a brief summary of our proof. Given a topological branched cover $\phi: \Sigma \rightarrow \mathbb{P}^{1}$, one may form the corresponding Hurwitz space $\mathcal{H}$, a moduli space whose points represent those branched covers $\Sigma \rightarrow \mathbb{P}^{1}$ which may be obtained from $\phi$ by moving around the images of the branch points in $\mathbb{P}^{1}$ while holding constant the combinatorial branch structure over these points as they move. Each of these branched covers gives rise to a complex structure on $\Sigma$ by pulling back the one on $\mathbb{P}^{1}$. This defines a map $\Psi: \mathcal{H} \rightarrow \mathcal{M}_{\Sigma}$, where $\mathcal{M}_{\Sigma}$ is the moduli space of complex structures on $\Sigma$. Under the assumption that $\phi: \Sigma \rightarrow \mathbb{P}^{1}$ is completely non-Galois (i.e., it has no non-trivial deck transformations), one may show that $\Psi$ lifts to a map $\widetilde{\Psi}: Q \rightarrow \mathcal{T}_{\Sigma}$, where $Q$ is a regular covering space of $\mathcal{H}$ and $\mathcal{T}_{\Sigma}$ is the Teichmuller space of $\Sigma$, and that $\widetilde{\Psi}$ is equivariant with respect to a natural group homomorphism $R: \operatorname{Deck}(Q \rightarrow \mathcal{H}) \rightarrow \Gamma_{\Sigma}$, where $\Gamma_{\Sigma}$ denotes the mapping class group of $\Sigma$ acting on $\mathcal{T}_{\Sigma}$. Our proof then proceeds as follows: We first prove an algebraic lemma enabling us to construct a topological branched cover $\phi: \Sigma \rightarrow \mathbb{P}^{1}$, which has the branch structure and monodromy specified in the theorem. We then show that for the corresponding Hurwitz space, $R$ has infinite image. Because every point of $\mathcal{T}_{\Sigma}$ has finite stabilizer in $\Gamma_{\Sigma}$, it follows that $\widetilde{\Psi}$ and hence $\Psi$ are nonconstant. Because $\Psi$ is an algebraic map between algebraic varieties, when $\mathcal{M}_{\Sigma}$ has dimension 1, $\Psi$ maps $\mathcal{H}$ onto a Zariski open subset of $\mathcal{M}_{\Sigma}$, i.e., onto a set with finite complement. This proves the theorem; given any genus one Riemann surface, elements of the inverse image of the corresponding point in $\mathcal{M}_{\Sigma}$ are branched covers with the desired property (since combinatorially, they are identical to $\phi$ ). Whenever the genus of $\mathcal{M}_{\Sigma}$ is positive, $\Psi$ is non-constant.

\section{A topological CONSTRUCTION OF THE BRANCHED COVERING}

We begin by reminding the reader how any given $n$-sheeted branched covering $\phi: \Sigma \rightarrow \mathbb{P}^{1}$ may be described combinatorially. Let $\left\{x_{1}, \ldots, x_{r}\right\} \subset \mathbb{P}^{1}$ denote the points over which branching occurs, and choose a basepoint $x_{0} \in \mathbb{P}^{1}$ disjoint from the other $x_{i}$ 's. Let $w_{1}, \ldots, w_{r}$ denote simple closed curves in $\mathbb{P}_{0}^{1}:=\mathbb{P}^{1}-\left\{x_{1}, \ldots, x_{r}\right\}$, all based at $x_{0}$, which satisfy (see Figure 1 ):

(1) Each $w_{i}$ bounds a disc $D_{i} \subset \mathbb{P}^{1}$ such that $D_{i} \cap\left\{x_{1}, \ldots, x_{r}\right\}=\left\{x_{i}\right\}$.

(2) If $i \neq j$, then $D_{i} \cap D_{j}=\left\{x_{0}\right\}$.

(3) Each $w_{i}$ is oriented counterclockwise as the boundary of $D_{i}$.

(4) $\prod_{i=1}^{r} w_{i}=1$ in $\pi_{1}\left(\mathbb{P}_{0}^{1}, x_{0}\right)$.

Label the points in $\phi^{-1}\left(x_{0}\right)$ by the numbers $\{1, \ldots, n\}$. Then each loop $w_{i}$ gives rise to a permutation $\rho_{i} \in S_{n}$, the symmetric group. Think of $\rho_{i}$ as acting on $\{1, \ldots, n\}$ from the right. Define a group homomorphism $\rho: \pi_{1}\left(\mathbb{P}_{0}^{1}, x_{0}\right) \rightarrow S_{n}$ by $\rho\left(w_{i}\right)=\rho_{i}$ (this $\rho$ is the monodromy representation of $\left.\phi\right)$. Define the signature of the branched cover $\phi: \Sigma \rightarrow \mathbb{P}^{1}$ to be the $n$-tuple of permutations $\left(\rho_{1}, \ldots, \rho_{r}\right)$. Conversely, suppose we are just given the points $\left\{x_{1}, \ldots, x_{r}\right\} \subset \mathbb{P}^{1}$, the loops $w_{1}, \ldots, w_{r}$ (as above), and the permutations $\rho_{1}, \ldots \rho_{r} \in S_{n}$ satisfying $\prod_{i=1}^{r} w_{i}=1$. Reconstruct the surface $\Sigma$ and the branched covering $\phi: \Sigma \rightarrow \mathbb{P}^{1}$ as follows. First construct the (unbranched) cover $\phi_{0}: \Sigma_{0} \rightarrow \mathbb{P}_{0}^{1}$ corresponding to $\rho$ using covering space theory. Then fill in one point for each end of $\Sigma_{0}$ to obtain $\Sigma$, and extend $\phi_{0}$ continuously to $\phi$ on $\Sigma$ in the only possible way. 


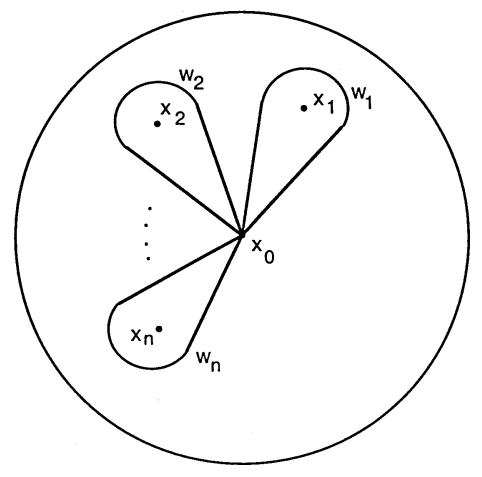

FIGURE 1.

Thus, to create a branched covering with certain properties, one needs to produce permutations with corresponding properties. Hence the following lemma:

Lemma 1. Let $n \geq 4$, and consider $\rho_{1}=(123)$ and $\rho_{2}=(132)$ in $S_{n}$. Then it is possible to choose $\rho_{3}, \ldots, \rho_{n} \in S_{n}$ such that:

(1) $\rho_{i}$ is a 3-cycle for each $i$.

(2) $\prod_{i=1}^{n} \rho_{i}=1$.

(3) The number "1" doesn't occur in any of the 3 -cycles $\rho_{3}, \ldots, \rho_{n}$ : all fix 1.

(4) The subgroup of $S_{n}$ generated by $\left\{\rho_{3}, \ldots, \rho_{n}\right\}$ acts transitively on $\{2, \ldots, n\}$.

(5) $\left\{\rho_{1}, \ldots, \rho_{n}\right\}$ generate $A_{n}$.

Proof. We will denote by $\vec{\rho}_{n}$ the $n$-tuple $\left(\rho_{1}, \ldots, \rho_{n}\right)$. Let

$$
\vec{\rho}_{4}=((123),(132),(234),(243))
$$

and

$$
\vec{\rho}_{5}=((123),(132),(234),(245),(253)) .
$$

It is easily verified that these signatures satisfy the five conditions specified in the lemma. Inductively, if $n>5$ define $\vec{\rho}_{n}$ by adjoining the permutations $\rho_{n-1}=$ $(2 n-1 n)$ and $\rho_{n}=(2 n n-1)$ to the $(n-2)$-tuple $\vec{\rho}_{n-2}$. It is an elementary exercise (which we omit) to show that $\vec{\rho}_{n}$ satisfies the conditions of the theorem for all $n$. This completes the proof of Lemma 1 .

Fix an $n \geq 4$, choose $n$ distinct points $x_{1}, \ldots, x_{n} \in \mathbb{P}^{1}$, a basepoint $x_{0} \in \mathbb{P}_{0}^{1}$, and $n$ based loops $w_{i}$ related to the $x_{i}$ 's as described above. Use the signature $\vec{\rho}_{n}$ produced in Lemma 1 to construct a branched cover $\phi: \Sigma \rightarrow \mathbb{P}^{1}$, branched over the $x_{i}$ 's. By construction, this $n$-sheeted cover will be connected and have monodromy group $A_{n}$. By the Riemann-Hurwitz formula, genus $(\Sigma)=1$.

\section{HuRWitz SPACES}

In this section we give a construction of the Hurwitz space corresponding to a branched cover. (Note: The definition of a Hurwitz space given in this paper corresponds to a single connected component of a Hurwitz space as defined in [F2].) We will start with a general finite-sheeted branched cover, and then specialize to the ones constructed in the last section. So, begin by letting $\phi: \Sigma \rightarrow \mathbb{P}^{1}$ be any $n$-sheeted branched cover, branched over $\left\{x_{1}, \ldots, x_{r}\right\}$. Let Homeo $\left(\mathbb{P}^{1}\right)$ denote the 
topological group of orientation preserving self-homeomorphisms of $\mathbb{P}^{1}$. Define the Hurwitz space $\mathcal{H}$ corresponding to the branched cover $\phi$ by

$$
\mathcal{H}=\left\{g \circ \phi: \Sigma \rightarrow \mathbb{P}^{1} \text { such that } g \in \operatorname{Homeo}\left(\mathbb{P}^{1}\right)\right\} / \sim
$$

where $g_{1} \circ \phi \sim g_{2} \circ \phi$ if and only if there exists a homeomorphism $h: \Sigma \rightarrow \Sigma$ such that the diagram

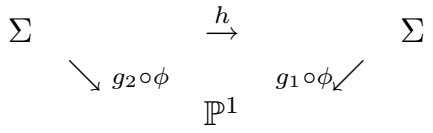

commutes. Note that $g_{1} \circ \phi \sim g_{2} \circ \phi$ if and only if there exists an $h \in \operatorname{Homeo}(\Sigma)$ such that $\left(g_{1}^{-1} g_{2}\right) \phi=\phi h$. Thus we may write $\mathcal{H} \cong \operatorname{Homeo}\left(\mathbb{P}^{1}\right) / G$, where $G \subset$ $\operatorname{Homeo}\left(\mathbb{P}^{1}\right)$ is the subgroup consisting of those homeomorphisms $g$ of $\mathbb{P}^{1}$ which lift to a homeomorphism $h_{g}$ of $\Sigma$ making the diagram

$$
\begin{array}{ccc}
\Sigma & \stackrel{h_{g}}{\rightarrow} & \Sigma \\
\phi \downarrow & & \phi \downarrow \\
\mathbb{P}^{1} & \stackrel{g}{\rightarrow} & \mathbb{P}^{1}
\end{array}
$$

commute. Let $S_{r}$ act on $\left(\mathbb{P}^{1}\right)^{r}$ by permuting the coordinates, and define $\Delta \subset\left(\mathbb{P}^{1}\right)^{r}$ by $\Delta=\left\{\left(y_{1}, \ldots, y_{r}\right) \in\left(\mathbb{P}^{1}\right)^{r}: y_{i}=y_{j}\right.$ for some $\left.i \neq j\right\}$. Define

$$
\Pi=\left(\left(\mathbb{P}^{1}\right)^{r}-\Delta\right) / S_{r} .
$$

Define a map $P: \operatorname{Homeo}\left(\mathbb{P}^{1}\right) \rightarrow \Pi$ by $P(f)=\left[f\left(x_{1}\right), \ldots, f\left(x_{r}\right)\right]$. Define the following two subgroups of Homeo $\left(\mathbb{P}^{1}\right)$ :

$$
\begin{aligned}
& \mathcal{G}=P^{-1}\left[x_{1}, \ldots, x_{r}\right], \\
& \mathcal{G}_{0}=\text { the identity component of } \mathcal{G} .
\end{aligned}
$$

We now observe that

$$
\mathcal{G}_{0} \subseteq G \subseteq \mathcal{G}
$$

The second of these inclusions is completely elementary; since $\phi \circ h$ and $g \circ \phi$ are two ways of writing the same branched cover, they must have the same branch locus in $\mathbb{P}^{1}$. Hence, $g\left[x_{1}, \ldots, x_{r}\right]=\left[x_{1}, \ldots, x_{r}\right]$.

To prove the first inclusion, $\mathcal{G}_{0} \subseteq G$, we quote two lemmas from $\mathrm{KlKo}$, Lemmas 2 and 3$]$ :

Lemma 2. If $r \geq 3$, then $\pi_{i}\left(\mathcal{G}_{0}\right)=0$ for all $i$.

We omit the proof of Lemma 2 ; the reader is referred to [KIKo].

Lemma 3. Given $g \in \mathcal{G}_{0}$, there is a homeomorphism $h_{g}: \Sigma \rightarrow \Sigma$ such that $g \circ \phi=$ $\phi \circ h_{g}$. If $r \geq 3$, then $h_{g}$ is uniquely determined by $g$ and, in fact, $g \mapsto h_{g}$ defines a continuous group homomorphism $\mathcal{G}_{0} \rightarrow \operatorname{Homeo}(\Sigma)$ such that $\phi$ is equivariant with respect to the resulting action of $\mathcal{G}_{0}$ on $\Sigma$.

Proof. Let $g \in \mathcal{G}_{0}$. Choose a path $g_{t}$ in $\mathcal{G}_{0}$ from the identity to $g$. Let $y \in \phi^{-1}\left(\mathbb{P}_{0}^{1}\right)$. Let $\alpha: I \rightarrow \phi^{-1}\left(\mathbb{P}_{0}^{1}\right)$ be the lift of the path $g_{t}(\phi(y))$ which starts at $y$, and define $h_{g}(y)=\alpha(1)$. Define $h_{g}$ to be the identity on $\phi^{-1}\left(\left\{x_{1}, \ldots, x_{n}\right\}\right)$. Then $h_{g}: \Sigma \rightarrow \Sigma$ is a homeomorphism and $g \circ \phi=\phi \circ h_{g}$. Furthermore, if $r \geq 3$, then, since $\pi_{1}\left(\mathcal{G}_{0}\right)=0$, any two such paths $g_{t}$ would lead to homotopic paths in $\mathbb{P}_{0}^{1}$. Hence, for $r \geq 3, g \mapsto h_{g}$ is a well-defined homomorphism $\mathcal{G}_{0} \rightarrow \operatorname{Homeo}(\Sigma)$ making $\phi$ equivariant. This completes the proof of Lemma 3. 
For the rest of this section assume that the branched cover $\phi: \Sigma \rightarrow \mathbb{P}^{1}$ is completely non-Galois: It has no non-trivial deck transformations. This is equivalent to the algebraic assumption that the monodromy group of $\phi$ has trivial centralizer in $S_{n}$. From this, we have a well-defined group homomorphism $G \rightarrow \operatorname{Homeo}(\Sigma)$ given by $g \mapsto h_{g}$, where $h_{g}$ is defined as in the definition of $G$.

Next, we construct some useful covering maps. Let $\Pi=\left(\left(\mathbb{P}^{1}\right)^{r}-\Delta\right) / S_{r}$, which is homeomorphic to $\operatorname{Homeo}\left(\mathbb{P}^{1}\right) / \mathcal{G}$ and let $Q=\operatorname{Homeo}\left(\mathbb{P}^{1}\right) / \mathcal{G}_{0}$. Because $\mathcal{G}_{0}$ is the identity component of $G$ and of $\mathcal{G}$, it follows that the natural quotient maps $Q \rightarrow$ $\mathcal{H} \rightarrow \Pi$ are both covering maps. Furthermore, since $\mathcal{G}_{0}$ is a normal subgroup of $G$, the covering map $Q \rightarrow \mathcal{H}$ is regular (Galois), with deck group equal to $G / \mathcal{G}_{0}$. This deck group acts on $Q$ from the right in the obvious manner, with quotient $\mathcal{H}$. Note that $Q$ is almost, but not quite, the universal cover of $\mathcal{H} ; \pi_{1}(Q)=\pi_{1}\left(\operatorname{Homeo}\left(\mathbb{P}^{1}\right)\right)=$ $Z_{2}$, since $\mathcal{G}_{0}$ is contractible and $S O(3) \rightarrow \operatorname{Homeo}\left(\mathbb{P}^{1}\right)$ is a homotopy equivalence (a fact dating back to Kneser $[\mathrm{K}]$ in 1926).

We now remind the reader of some basic Teichmuller theory. Given the closed oriented (topological) surface $\Sigma$, define the Teichmuller space $\mathcal{T}_{\Sigma}$ by

$$
\mathcal{T}_{\Sigma}=\left\{\left(\Sigma_{0},\left[q_{0}\right]\right): \Sigma_{0}\right. \text { is a Riemann surface and }
$$

$\left[q_{0}\right]$ is an isotopy class of homeomorphisms $\left.\Sigma \rightarrow \Sigma_{0}\right\} / \sim$

where we define $\left(\Sigma_{0}, q_{0}\right) \sim\left(\Sigma_{1}, q_{1}\right)$ if there is an analytic isomorphism $h: \Sigma_{0} \rightarrow \Sigma_{1}$ such that $q_{1} \circ h$ is isotopic to $q_{0}$.

The mapping class group of $\Sigma$, defined by $\Gamma_{\Sigma}=\operatorname{Homeo}(\Sigma) /$ isotopy, acts on $\mathcal{T}_{\Sigma}$ from the right by

$$
\left(\Sigma_{0},\left[q_{0}\right]\right) \cdot[h]=\left(\Sigma_{0},\left[q_{0} \circ h\right]\right) .
$$

The quotient of $\mathcal{T}_{\Sigma}$ under this action is the moduli space of $\Sigma$, defined by

$\mathcal{M}_{\Sigma}=\left\{\right.$ Riemann surfaces $\Sigma_{0}$ homeomorphic to $\left.\Sigma\right\}$ /analytic isomorphism.

Let $p: \Sigma \rightarrow \mathbb{P}^{1}$ be any branched cover; define $\Sigma_{p}$ to be the Riemann surface with underlying space $\Sigma$ and with the unique complex structure making $p$ analytic. We now define maps $\Psi: \mathcal{H} \rightarrow \mathcal{M}_{\Sigma}$ and $\widetilde{\Psi}: Q \rightarrow \mathcal{T}_{\Sigma}$ by $\Psi(f G)=\Sigma_{f \phi}$ and $\widetilde{\Psi}\left(f \mathcal{G}_{0}\right)=\left(\Sigma_{f \phi}, \mathrm{id}_{\Sigma}\right)$. It is immediately clear that the following diagram commutes:

$$
\begin{array}{ccccc}
\text { Homeo }\left(\mathbb{P}^{1}\right) / \mathcal{G}_{0} & = & Q & \stackrel{\widetilde{\Psi}}{\rightarrow} & \mathcal{T}_{\Sigma} \\
\downarrow & & \downarrow & & \downarrow \\
\text { Homeo }\left(\mathbb{P}^{1}\right) / G & = & \mathcal{H} & \stackrel{\Psi}{\rightarrow} & \mathcal{M}_{\Sigma}
\end{array}
$$

The vertical arrows in this diagram are simply quotient maps involving the right action of $G / \mathcal{G}_{0}$ on $Q$ and the right action of $\Gamma_{\Sigma}$ on $\mathcal{T}_{\Sigma}$. Define a group homomorphism $R: G / \mathcal{G}_{0} \rightarrow \Gamma_{\Sigma}$ by $g \mathcal{G}_{0} \mapsto\left[h_{g}\right]$. The fact that $R$ is well-defined follows from the proof of Lemma 3, which actually shows that if $g \in \mathcal{G}_{0}$, then $h_{g}$ is homotopic (hence isotopic) to the identity. In [KlKo] we give a general algorithm for computing the composition of $R$ with the natural homomorphism $\Gamma_{\Sigma} \rightarrow S L(2 g, \mathbb{Z})$ (defined by action on $H_{1}(\Sigma)$ ). In the genus one case, this gives $R$ precisely, since $\Gamma_{\Sigma} \rightarrow S L(2, \mathbb{Z})$ is an isomorphism. In the current paper, instead of using this general method, we get the information we need from a specific geometric observation in the next section.

Lemma 4. $\widetilde{\Psi}$ is equivariant with respect to the homomorphism $R: G / \mathcal{G}_{0} \rightarrow \Gamma_{\Sigma}$. 
Proof. We need to show that if $f \in \operatorname{Homeo}\left(\mathbb{P}^{1}\right)$ and $g \in G$, then $\widetilde{\Psi}\left(f \mathcal{G}_{0} \cdot g\right)=$ $\left(\widetilde{\Psi}\left(f \mathcal{G}_{0}\right)\right) \cdot\left[h_{g}\right]$. Restating using the definitions, we need to show that $\left(\Sigma_{f g \phi},[i d]\right) \sim$ $\left(\Sigma_{f \phi},\left[h_{g}\right]\right)$. In other words, we need to show that the diagram

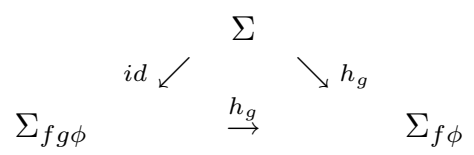

commutes up to homotopy (which is obvious!), and that $h_{g}: \Sigma_{f g \phi} \rightarrow \Sigma_{f \phi}$ is analytic. To prove this second fact, consider the diagram

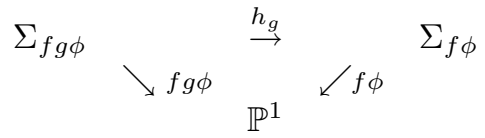

which commutes by definition of $h_{g}$. The two vertical branched cover maps are analytic by definition of the complex structures on the $\Sigma$ 's. Conclude the homeomorphism $h_{g}$ is analytic as well. This completes the proof of Lemma 4 .

\section{Statement and Proof of Theorem 1}

Theorem 1. Let $n \geq 4$ be an integer. There exists a finite subset $Y \subset \mathcal{M}_{1}$ (where $\mathcal{M}_{1}$ is the moduli space of genus one Riemann surfaces) with the following property. If $\Sigma_{0}$ is a Riemann surface of genus one, and $\left[\Sigma_{0}\right] \notin Y$, then there exists a holomorphic function $f: \Sigma_{0} \rightarrow \mathbb{P}^{1}$ of degree $n$ such that all branch points of $f$ have multiplicity 3 , no two branch points of $f$ map to the same point in $\mathbb{P}^{1}$, and the monodromy group of $f$ is the full alternating group $A_{n}$.

Proof. Fix $n$. Let $\phi: \Sigma \rightarrow \mathbb{P}^{1}$ be the topological branched cover with monodromy group $A_{n}$ constructed in Section 2 using Lemma 1. In building this cover, we may choose our branch points $x_{1}, \ldots, x_{n}$ and our basepoint $x_{0}$ arbitrarily in $\mathbb{P}^{1}$. Since $A_{n}$ has trivial centralizer in $S_{n}$, the branched cover $\phi: \Sigma \rightarrow \mathbb{P}^{1}$ is completely non-Galois, and hence we can use $\phi$ to make all the constructions of Section 3 involving Hurwitz spaces, Teichmuller theory, etc. Express $\mathbb{P}^{1}$ as the union of two discs $B_{1}$ and $B_{2}$ whose intersection and common boundary is a smooth circle $C$. Choose these discs so that $B_{1}$ contains $D_{1} \cup D_{2}, B_{2}$ contains $D_{3} \cup \cdots \cup D_{n}$ and, for $i=1, \ldots, n, C \cap D_{i}=x_{0}$. See Figure 2 .

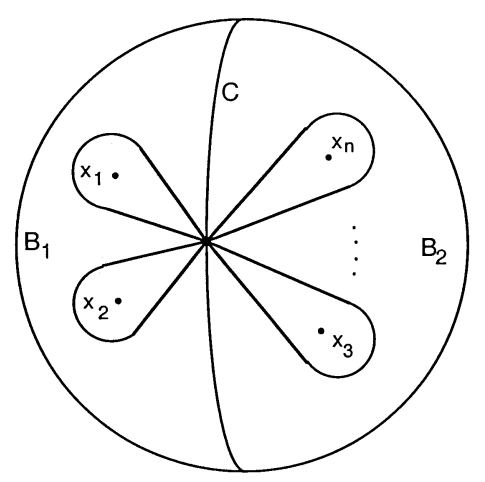

Figure 2. 
We now wish to visualize the topology of $\phi^{-1}\left(B_{1}\right)$ and $\phi^{-1}\left(B_{2}\right)$. The monodromy along the curve $C$ is trivial: $\rho_{1} \rho_{2}$ is the identity. Conclude that $\phi^{-1}(C)$ consists of $n$ disjoint circles, each mapped homeomorphically to $C$ by $\phi$. Since we numbered the points of $\phi^{-1}\left(x_{0}\right)$ using $\{1, \ldots, n\}$, this allows labelling components of $\phi^{-1}(C)$ as $C_{1}, \ldots, C_{n}$ according to which point of $\phi^{-1}\left(x_{0}\right)$ they contain. Using the algebraic properties of $\rho_{1}, \ldots, \rho_{n}$ in Lemma 1 , conclude the following: $\phi^{-1}\left(B_{1}\right)$ consists of one component with boundary $C_{1} \cup C_{2} \cup C_{3}$ and $n-3$ other components; each of these other components has as its boundary one of the remaining $C_{i}$ 's (for $i>3$ ), and is mapped homoemorphically onto $B_{1}$. On the other hand, $\phi^{-1}\left(B_{2}\right)$ consists of only two components: the first maps homeomorphically to $B_{2}$ and has as its boundary $C_{1}$ and the second has as its boundary $C_{2} \cup \cdots \cup C_{n}$. We illustrate this situation in Figure 3, with $\mathbb{P}^{1}$ and $\Sigma$ shown split in two along $C$ and $\phi^{-1}(C)$.

Let $A \subset B_{1}$ be a thin collar of $C=\partial B_{1}$, i.e., an annulus in $B_{1}$ one of whose boundary components is $C$. Define $g \in \operatorname{Homeo}\left(\mathbb{P}^{1}\right)$ to be a single Dehn twist along $A$. More precisely, the Dehn twist $g$ is defined as follows. Identify $A$ with $S^{1} \times[0,1]$ and define $g: A \rightarrow A$ by $g(z, t)=\left(e^{2 \pi i t} z, t\right)$. Clearly, $g$ is a homeomorphism of $A$ which is the identity on $\partial A$. Extend $g$ to all of $\mathbb{P}^{1}$ by defining it to be the identity outside of $A$. If we define $h_{g} \in \operatorname{Homeo}(\Sigma)$ to consist of simultaneous Dehn twists along all $n$ components of $\phi^{-1}(A)$, then $\phi \circ h_{g}=g \circ \phi$. We conclude that $g \in G$ and $R\left(g \mathcal{G}_{0}\right)=\left[h_{g}\right]$. Referring to Figure 3 , note that all the $C_{i}$ 's except $C_{2}$ and $C_{3}$ bound discs in $\Sigma\left(C_{1}\right.$ bounds a disc in $\phi^{-1}\left(B_{2}\right)$ while $C_{4}, \ldots, C_{n}$ bound discs

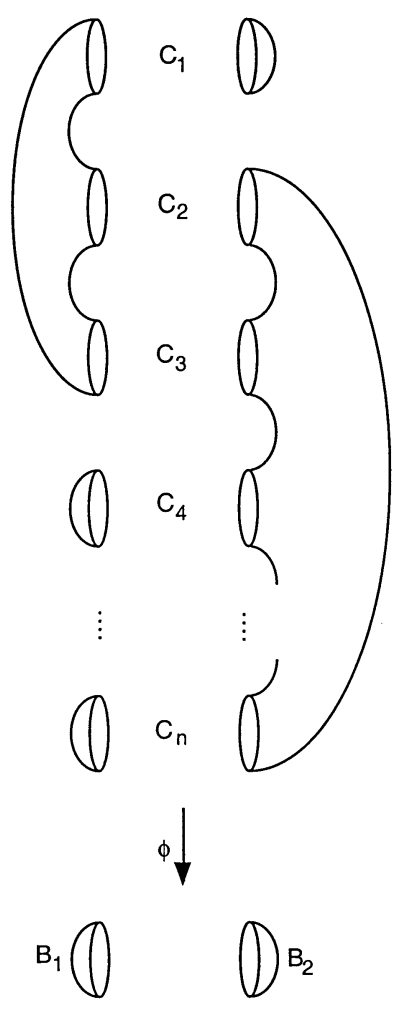

FIGURE 3. 
in $\left.\phi^{-1}\left(B_{1}\right)\right)$; hence the corresponding Dehn twists are trivial in the mapping class group $\Gamma_{\Sigma}$. The curves $C_{2}$ and $C_{3}$ are isotopic to each other in $\Sigma$ (by inspection of Figure 3 ); hence their Dehn twists are equal to each other in $\Gamma_{\Sigma}$. We conclude that $\left[h_{g}\right]$ is a double Dehn twist along the essential curve $C_{2}$ in the torus $\Sigma$. Hence $\left[h_{g}\right]$ is of infinite order in $\Gamma_{\Sigma}$ : That a Dehn twist along an essential curve in a closed orientable surface has infinite order in the mapping class group follows easily by considering its action on the fundamental group. Since each point in $\mathcal{T}_{\Sigma}$ has finite stabilizer in $\Gamma_{\Sigma}$, it follows that $\widetilde{\Psi}: Q \rightarrow \mathcal{T}_{\Sigma}$ and, hence, $\Psi: \mathcal{H} \rightarrow \mathcal{M}_{\Sigma}$ are nonconstant functions. Since $\mathcal{H}$ and $\mathcal{M}_{\Sigma}$ both have the structure of quasiprojective varieties (see [M], p. 25 , for $\mathcal{M}_{g}$ and [F1], p. 53 , for $\mathcal{H}$ ), $\Psi$ is an algebraic map which extends to the compactification of $\mathcal{H}$ (see $\mathrm{Gr}$, p. 247). When $\mathcal{M}_{\Sigma}$ has dimension 1 ( $\Sigma$ has genus one), conclude that the image of $\mathcal{H}$ in $\mathcal{M}_{\Sigma}$ is a quasiprojective subvariety of dimension one. Hence $\mathcal{M}_{\Sigma}-\Psi(\mathcal{H})$ consists of at most a finite number of points. This finishes the proof of Theorem 1.

Comment 0. F3 shows, for each $r \geq n, \mathcal{H}$ has exactly two components. Further, the Riemann surfaces parametrized by each have branch cycles satisfying the conditions of Lemma 1. In particular, $\Psi$ restricted to each component is non-constant.

Comment 1. We originally conceived of Theorem 1 as an application of Fried's Theorem 3.6 in [F2], which states that if a certain representation of $\pi_{1}(\mathcal{H})$ on $H_{1}(\Sigma ; \mathbb{Z})$ has infinite image, then $\Psi$ is non-constant. However, one gets a similar result by considering our homomorphism $R$ instead, which is a natural lift of Fried's representation. In addition, $R$ has infinite image by the pictorial argument involving Dehn twists given here, rather than by the more algebraic computations involving $H_{1}(\Sigma ; \mathbb{Z})$ (see for example $[\mathrm{F} 2]$ and $[\mathrm{KlKo}]$ ). We present this view for the sake of variety, and because we think it may appeal to the more geometrically-minded reader.

Comment 2. Having proved that, for each $n \geq 4$, the map $\Psi: \mathcal{H} \rightarrow \mathcal{M}_{1}$ misses at most a finite number of points of $\mathcal{M}_{1}$, it is natural to ask, for each such $n$, whether the map does in fact miss some points or whether it might actually be surjective. Mark van Hoeij, using very nice computations involving $J$-invariants, has shown that in the case $n=5$ the map $\Psi$ is actually surjective. For higher values of $n$, it seems likely that it remains surjective but someone needs to prove it! For $n=4$, we don't have a conjecture.

Comment 3. The preprint [F3] makes further applications of Dehn twists in order to compute explicitly the monodromy action of $\pi_{1} Q$ on the cohomology of a Riemann surface corresponding to a point on a Hurwitz space. (For other examples of this, see [F2] and [KlKo.) As a result, the map $\Psi$ is shown in [F3] to have higher dimensional image on other components of Hurwitz spaces constructed from $r$-tuples of 3-cycles corresponding to higher genus covers of $\mathbb{P}^{1}$. We don't, however, know this dimension as a precise function of $r$ and $n$.

\section{REFERENCES}

[F1] Fried, M., Fields of definition of function fields and Hurwitz families, Comm. in Alg. 5(1) 1977, 17-82. MR 56:12006

[F2] Fried, M., Combinatorial computations of moduli dimension of Nielsen classes of covers, Cont. Math., vol. 89, 1989, 61-79. MR 90j:12007

[F3] Fried, M., Alternating Groups and Lifting Invariants, preprint, 1999. 
[Gr] Griffiths, P., Periods of integrals on algebraic manifolds: summary of main results and discussion of open problems, Bull. AMS, Mar. 1970, 228-296. MR 41:3470

[GN] Guralnick, R. and Neubauer, M., Recent developments in the inverse Galois problem, Cont. Math., vol.186, (1996), 325-352. [MR 96c:00033

[KlKo] Klassen, E. and Kopeliovich, Y., Hurwitz spaces and braid group representations (1997), preprint.

[K] Kneser, H., Die Deformatiofnssatze der einfach zusammenhangenden Flachen, Math. Zeit., vol. 25 (1926) 362-372.

[M] Mumford, D., Curves and Their Jacobians, University of Michigan Press (1976). MR 54:7451

Department of Mathematics, University of California at Irvine, Irvine, California 92717

E-mail address: mfried@math.uci.edu

Department of Mathematics, Florida State University, Tallahassee, Florida 32306

E-mail address: klassen@math.fsu.edu

Unigraphics Solutions, 100824 Hope St., Cypress, California 90630

E-mail address: YKopeliovich@mail101.webango.com 